\title{
Label free cell tracking in 3-D tissue engineering constructs with high resolution imaging
}

\author{
W. A. Smith*a , K.-P. Lam ${ }^{a}$, K.P. Dempsey ${ }^{a}$, D. Mazzocchi-Jones ${ }^{b}$, J. B. Richardson ${ }^{c}$, Y. Yang ${ }^{b}$ \\ ${ }^{a}$ School of Computing and Mathematics, Keele University, Keele, Staffordshire, UK; ${ }^{b}$ Institute for \\ Science \& Technology in Medicine, Staffordshire, UK, ${ }^{\mathrm{c}}$ The Robert Jones and Agnes Hunt \\ Orthopaedic Hospital NHS Foundation Trust, Oswestry, UK
}

\begin{abstract}
Within the field of tissue engineering there is an emphasis on studying 3-D live tissue structures. Consequently, to investigate and identify cellular activities and phenotypes in a 3-D environment for all in vitro experiments, including shape, migration/proliferation and axon projection, it is necessary to adopt an optical imaging system that enables monitoring 3-D cellular activities and morphology through the thickness of the construct for an extended culture period without cell labeling. This paper describes a new 3-D tracking algorithm developed for Cell-IQ ${ }^{\circledR}$, an automated cell imaging platform, which has been equipped with an environmental chamber optimized to enable capturing time-lapse sequences of live cell images over a long-term period without cell labeling. As an integral part of the algorithm, a novel auto-focusing procedure was developed for phase contrast microscopy equipped with 20x and 40x objectives, to provide a more accurate estimation of cell growth/trajectories by allowing 3-D voxels to be computed at high spatiotemporal resolution and cell density. A pilot study was carried out in a phantom system consisting of horizontally aligned nanofiber layers (with precise spacing between them), to mimic features well exemplified in cellular activities of neuronal growth in a 3-D environment. This was followed by detailed investigations concerning axonal projections and dendritic circuitry formation in a 3-D tissue engineering construct. Preliminary work on primary animal neuronal cells in response to chemoattractant and topographic cue within the scaffolds has produced encouraging results.
\end{abstract}

Keywords: depth from focus, topographic surface, reconstruction, 2.5D imaging, multiscale, phase contrast microscopy, neuron proliferation

\section{INTRODUCTION}

Phase contrast microscopy is often used when label-free conditions are desired on culturing live cells. Capturing more detailed structures is reliant upon the usage of higher magnifications, which result in a decreased depth-of-view (DOV). This gives rise to the loss of focus of structures that exist outside this DOV. To address issue, multiple images along the $z$-direction may be captured, thus extending the depth of view in accord with the number of images captured. A suitable depth-from-delfocus algorithm must be applied in order to estimate the focus measures of the pixels within each image. The $(x, y, z)$ positions of the most in focus pixels are used to form a depth map that details the surface geometry of the 3$\mathrm{D}$ environment being imaged. The collective $z$-stack of images (Figure 1) may then be used to reconstruct the all-infocus image based on these in-focus pixel locations.

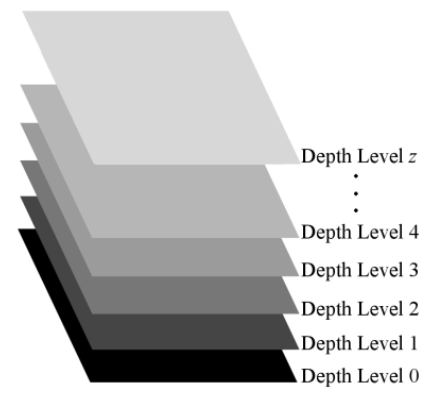

Figure 1: An illustration of the acquired $z$-stack of images. The camera within the CellIQ ${ }^{\circledR}$ captures the center $z$-plane $z / 2$ first, followed by sequentially capturing $z$-planes down to depth level 0 . The camera then captures depth level $z / 2+1$ up to depth level $z$. Each $z$-plane is in focus at that location in the sample, which therefore means that capturing the $z$-stack extends the DOV of the camera to $z^{*} D O V$. 


\subsection{Phase Contrast Imaging}

Phase Contrast Imaging is a well-established label-free, minimally-manipulative imaging technique [3]. The phase differences observed as light passes through changing mediums may be amplified using a phase plate, causing destructive interference between light originating from the initial source and light that has propagated through a cell or any other object within the field of view $[15,16]$. The disadvantage of this technique is that the signal-to-noise ratio is relatively low and it is also not capable of quantifying information such as specific protein expression within a cell culture. The advantage of this technique is that there is minimal manipulation of the cells compared with more invasive techniques such as histological analysis [4].

Unlike phase contrast microscopy, Fluorescence microscopy offers valuable information for studies that seek information regarding specific protein distribution within a culture of cells. This is particularly useful for identifying the relationship between cell behaviors and the presence (or absence) of particular proteins [5]. Confocal microscopy offers a higher spatial resolution, which is achieved by exciting flurophores only when they are in the focal plane of the microscope. This permits the identification and measurement of cell structure such as smooth muscle cells [2]. The disadvantage of the fluorescence and confocal techniques is that the duration of the experiment is limited to the amount of excitation that the markers used may undergo before photobleaching degrades them [1]. In addition, the usage of flurophores results in currently unquantifiable effects on the cells behavior and other characteristics, e.g. axon projection in live cell culture, which makes them unsuitable for applications in clinical therapy. It is therefore preferable to use the phase contrast technique over fluorescence microscopy techniques for extended studies of cell behaviors due to the marker-free requirements, ease of sample preparation and the relatively low levels of light exposure necessary to image the cell. The CellIQ ${ }^{\circledR}[13]$ phase contrast microscope suite is used for this study due to the built in automated $\mathrm{CO}_{2}$ gas and environmental temperature regulation.

\subsection{Depth from De/Focus}

Depth from de/focus algorithms [9] rely on the principle that the Gaussian blurring of an out of focus object can be modeled such that the point spread function (psf) may be estimated [8]. By capturing two or more images it may be possible to use this information to identify the distance between the lens and the object. Increasing the number of images captured should cause a proportionate increase the resolution of the distance measurements.

The technique employed in this study is a multiscale approach based on the minimum description length $(M D L)$ principle [7]. The aim of the $M D L$ principle is to describe the underlying system with the minimal complexity possible, which in this case is the optical configuration. In this study, the object is projected into defocused images depending on the distance from the lens objective. These distances influence the observation of details on different scales; the Gaussian blurring causes a greater loss of information on local features such as the cell membrane, than on global features such as the cultures general topography $[10,11]$. This blurring increases proportionately with the objects distance from the depth-of-view of the lens. This effect can be quantified using the multiscale approach by calculating the focus measures of each pixel based on the amount of information they possess. Here, the measure of information is defined as:

$$
D L=\lambda / \sigma+\varepsilon \quad(1)
$$

Where the description length $(D L)$ corresponds to the number of planes $(\lambda)$ divided by the $p s f(\sigma)$ in addition to the residual $(\varepsilon)$. The sigma value is based upon the distance of the object from the lens, which determines the degree to which the $p s f$ projects the defocussed image. Each pixel has a corresponding $D L$ calculated by (1), and the minimum $D L$ value is sought for each $\mathrm{x}, \mathrm{y}$ location along the z-axis. These $(\mathrm{x}, \mathrm{y}, \mathrm{z})$ locations form a depth map, which is used to reconstruct an all-in-focus image from the original z-stack of images.

\subsection{Paper Outline}

The paper is arranged as follows: Section 2 describes the experimental design and rationale for both the phantom experiments and the neuron growth preliminary study, Section 3 details the experiment results and Section 4 discusses the implications of the findings. Section 5 concludes the work and provides future directions for the studies. 


\section{EXPERIMENTAL DESIGN AND METHODS}

A CellIQ ${ }^{\circledR}$ microscope was used to capture the images for both experiments. The lenses used to image the phantom are a NIKON CFI Plan Fluor ELWD ADL 40XC NA 0.6, working distance $(W D) .3 .7-2.7 \mathrm{~mm}$ and a NIKON CFI S Plan Fluor ELWD ADM 20XC, NA 0.50, WD 8.2-6.9 mm. The CellIQ ${ }^{\circledR}$ machine motor can move at a minimum distance of $0.4 \mu \mathrm{m}$ per depth level image. The maximum size of the $z$-stack is $80 \mu \mathrm{m}$ due to movement limitations of the camera motor. This $z$-stack size was sufficient for the purposes of the experiments.

\subsection{Phantom Experiment Design}

A $2 \%(\mathrm{w} / \mathrm{v})$ poly-L,D-lactic acid (PLA) solution in mixture solvent of chloroform and dimethylformamide (7:3 solvent volume) was used to fabricate aligned nanofibres by electrospinning technique. A novel parallel electrode collector was used to obtain nanofibers in a highly aligned conformation [14]. The resulted low line density nanofibers were then mounted onto acetate frames and affixed using a spray adhesive, to be handleable/portable and to maintain their aligned conformation for use in following experiments. Nanofibers were desiccated overnight and sterilized in a UV chamber before use in cell culture experiments. The nanofiber diameter and line density have been measured by SEM images. The average fiber diameter was $534 \mathrm{~nm}$ and line density was $280 \mathrm{fibres} / \mathrm{mm}$. A typical SEM picture of the nanofiber is shown in Figure 2. The thickness of the electroformed copper mesh was $12.5 \mu \mathrm{m}$, with a $20 \%$ margin of error. The final structure of the phantom is illustrated in Figure 3.

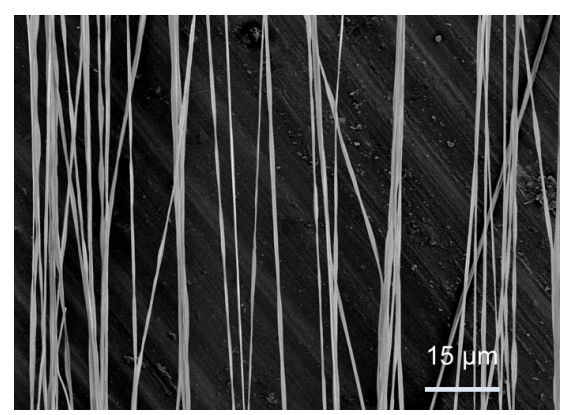

Figure 2: A SEM image of the electrospun nanofibers. The highly parallel structure of the layers allows their z-planes to be identified when orthogonally aranged along the z-axis.

The geometry of this phantom structure was then inspected using a CellIQ ${ }^{\circledR}$ phase contrast microscope. The known fiber and copper mesh measurements were compared to the details estimated by the $M D L /$ multiscale algorithm. In addition to this algorithm, two measures were used to identify highly focused z-planes within the $z$-stack, which were compared to the locations of structures within the estimated depth map. The Normalized Variance ( $N V)$ is one such statistic used to measure the focus of the $z$-stack of images [12]. The $N V$ is defined as: $N V=\frac{1}{X \times Y \times \mu} \times \sum_{X} \sum_{Y}(I(x, y)-\mu)$, where $\mathrm{X}$ and $\mathrm{Y}$ represent the height and width respectively of the image, $I$, and $\mu$ represents the mean image intensity. In addition to the $N V$, the Spatial Frequency $(S F)$ [6] was also used to identify highly focused images within the $z$-stack, based on the assumption that such images would possess a high degree of pixel intensity variation. I In order to calculate the $S F$, the Row Frequency $(R F)$ and Column Frequency $(C F)$ are calculated. Here, $R F$ is defined as: $R F \sqrt{\frac{1}{X \times Y} \sum_{x=0}^{x-1} \sum_{y=1}^{y-1}[I(x, y)-I x, y-1]^{2}}$ and CF is defined as: $C F \sqrt{\frac{1}{X \times Y} \sum_{y=0}^{y-1} \sum_{y=1}^{x-1}[I(x, y)-I(x-1, y)]^{2}}$. The SF is then calculated as: $S F=\sqrt{(R F)^{2}+(C F)^{2}}$. 


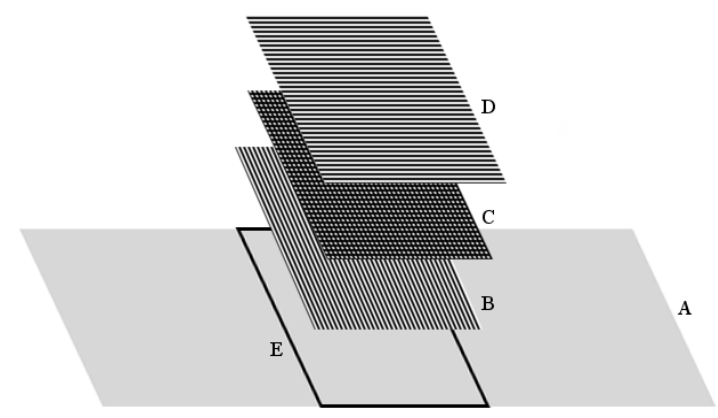

Figure 3: A diagram showing the configuration of the phantom sample. A nanofiber layer (B) is placed onto the microscope slide (A). This is followed by a copper mesh layer (C) and a second, orthogonally arranged nanofiber layer (D). Adhesive is then used to secure the layers (E).

\subsection{Neurite Outgrowth Experiment Method}

For cell culture experiments, the similar nanofiber assemble as in the phantom system has been used. Two aligned nanofiber meshes were mounted on round glass coverslip $(\sim 14 \mu \mathrm{m})$ by silicon gel in orthogonal arrangement with Al coil strip as spacer $(12 \mu \mathrm{m})$ (Figure 4). The assembled nanofiber scaffolds were sterilized by UV and then coated by polylysine $(50 \mu \mathrm{g} / \mathrm{ml})$ for overnight and laminin $(50 \mu \mathrm{g} / \mathrm{ml})$ for 4 hour. E19 rat embryos were isolated from placenta. The middle brain was dissected out and hippocampus region tissue was collected. The hippocampus cells were isolated out by application of trypsin/EDTA for 15 minutes. 10,000 hippocampus cells per scaffold have been seeded with neurobasal medium supplemented by B27, L-glutamin and antibiotics. After culturing in normal incubator at $37^{\circ} \mathrm{C}$ and $5 \% \mathrm{CO}^{2}$ for 4 days, the samples were then transferred to CellIQ ${ }^{\circledR}$ system for further culture for two days while multiple location and time point images were taken. The $z$-stack upper limit of $80 \mu \mathrm{m}$ was factored into the topographical design of the experiment, with the nanofiber layers and cells positioned within this range.

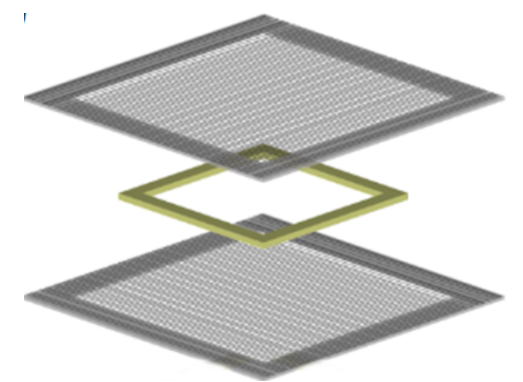

Figure 4: A diagram illustrating the aligned nanofiber meshes (upper and lower planes) and spacer (center plane) used for the cell culture experiment.

\section{PHANTOM EXPERIMENT RESULTS}

\subsection{Phantom sample depth values obtained using a 40x lens objective}

The height indexes (Figure 5) for the lower layer of nanofibers are within the 26-27 depth level region, while the upper layer nanofibers are estimated to be within the 82-88 depth level region, which corroborates with the position estimated by the focus measures (Figure 6). This yields a height difference of 58.5 depth levels or $23.4 \mu \mathrm{m}$, based on the average index values of the pixels corresponding to nanofibers. This gives a discrepancy of $10.1 \mu \mathrm{m}$ between the measured phantom size and the physical size identified in Figure 3. 


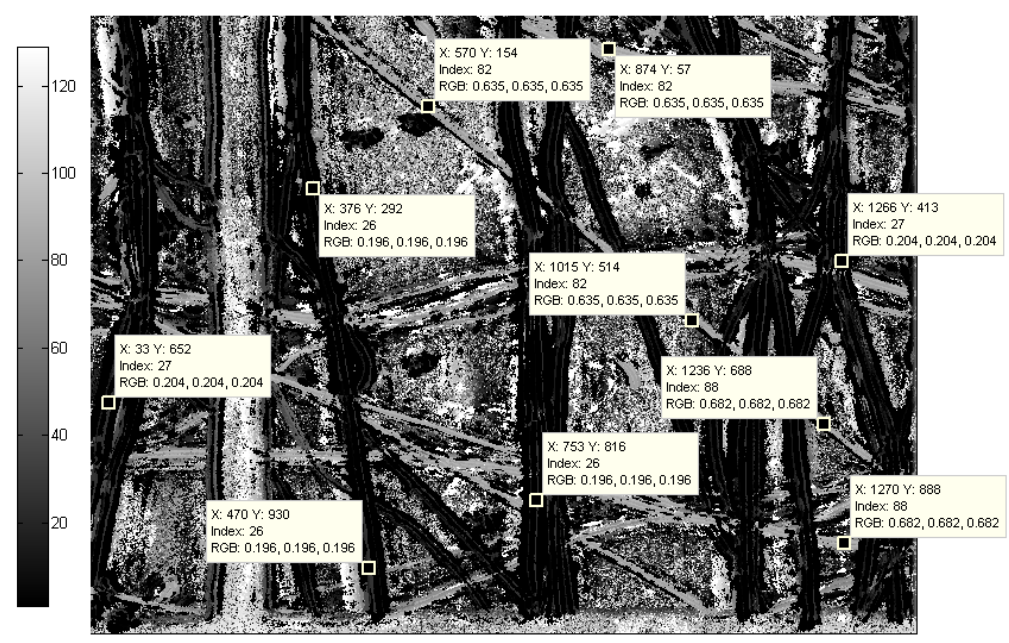

Figure 5: Depth map of a region within the phantom sample obtained using a 40x lens objective. The upper (A) and lower (B) nanofiber meshes have a separation distance of $23.4 \mu \mathrm{m}$. There are inaccuracies in the copper mesh region (C); however, this is understandable given that phase contrast imaging cannot resolve opaque or occluded objects.
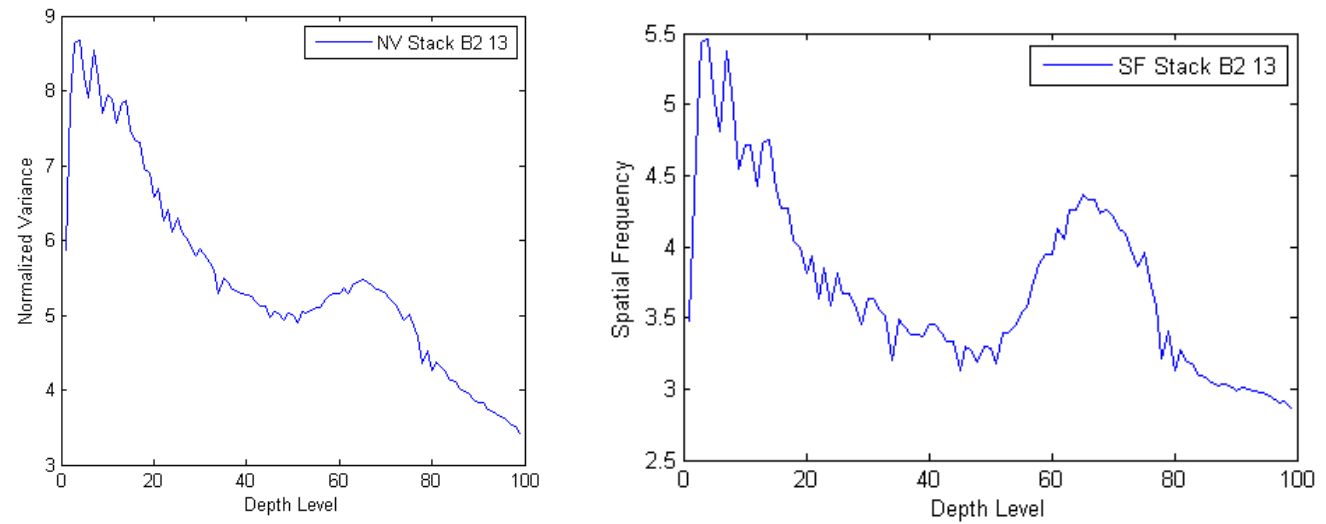

Figure 6: The Normalized Variance (left) and Spatial Frequency (right) of the z-stack. The two different measures identified similar depth level regions as being highly focused, however, the lower nanofiber layer is not as easy to identify compared with the upper layer.

\subsection{Phantom depth values obtained using a 20x lens objective}

The distance between the upper and lower nanofiber layers obtained using the 20x lens magnification is $17 \mu \mathrm{m}$ based on the depth map (Figure 7) with the upper nanofiber layer identified by the focus measures (Figure 8). This gives a discrepancy of $6.4 \mu \mathrm{m}$ between the $20 \mathrm{x}$ and $40 \mathrm{x}$ lens objectives. This difference may be accounted for by the margin of error in the mesh parameters $(0.8 \mu \mathrm{m})$ and the thickness increase from at least 2 overlapping nanofibers $(\sim 1 \mu \mathrm{m})$, as well as minor air spaces. 


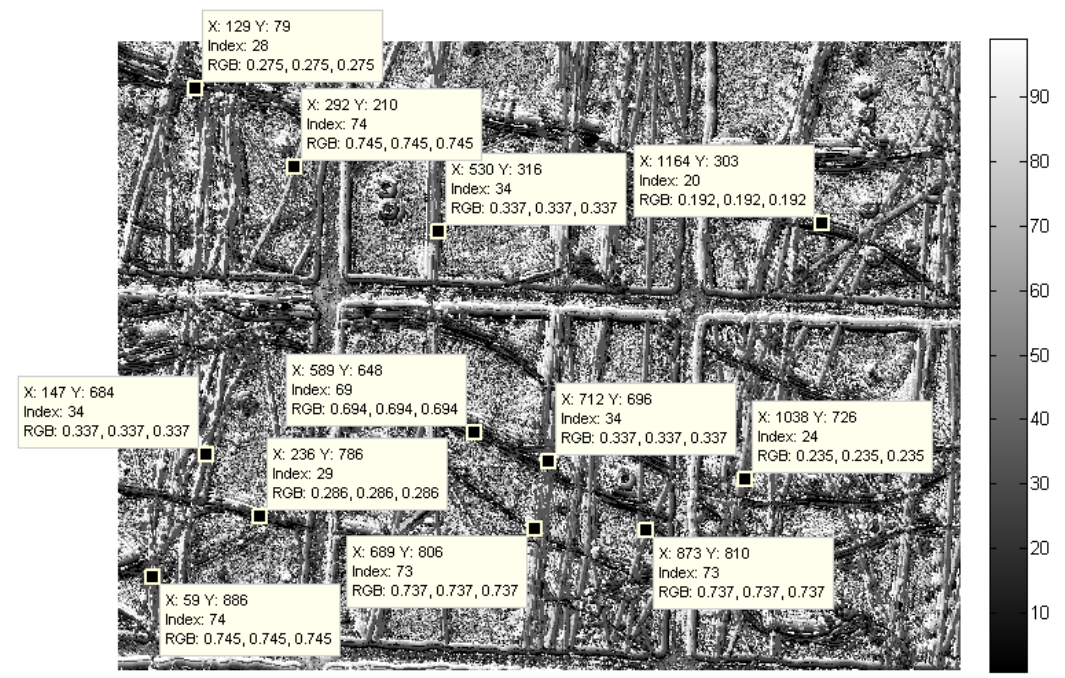

Figure 7: The depth map of the phantom data imaged under a 20x magnification lens in the region B2 06. The upper nanofiber layer is estimated to be located within the 69-74 depth level region, while the lower nanofiber layer is estimated to be located within the 24-34 depth level region. Based on the average upper and lower nanofiber positions, there is an estimated separation of 42.5 depth levels between the two layers, which corresponds to a distance of $17 \mu \mathrm{m}$.
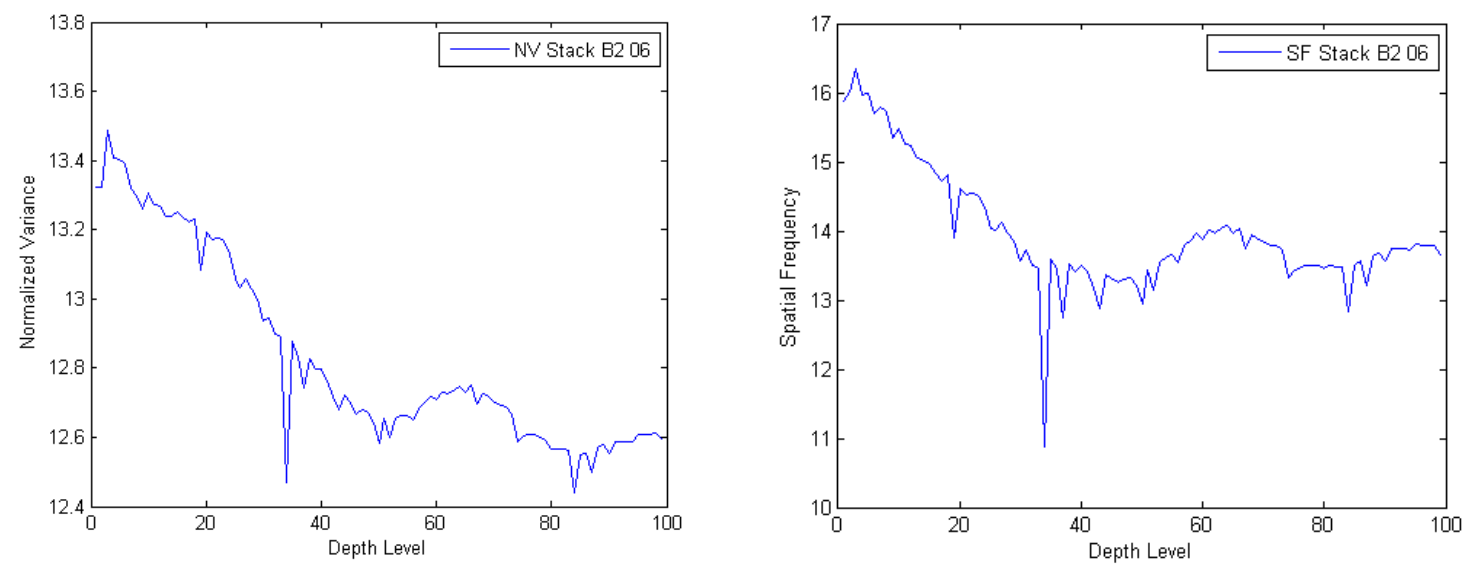

Figure 8: The Normalised Variance (left) and Spatial Frequency (Right) of the phantom sample identified in Figure 6. The minor peak in the 55-70 depth level region matches the position of the upper nanofiber layer estimated by the multiscale depth map. The lower nanofiber position is more difficult to discern, which may be due to the proximity of the layer to the copper mesh.

Table 1: A table summarizing the depth estimation algorithm findings. The sample thickness based on the 20x and 40x lens magnifications were averaged over 3 different ROIs and had a standard deviation of $2.1 \mu \mathrm{m}$.

\begin{tabular}{|l|l|}
\hline Magnification & $\begin{array}{l}\text { Mean Depth Estimation } \\
\text { Distance }(\mu \mathrm{m})\end{array}$ \\
\hline $20 \mathrm{x}$ & 21.6 \\
\hline $40 \mathrm{x}$ & 23.4 \\
\hline
\end{tabular}

\section{NEURITE OUTROWTH PRELIMINARY RESULTS}

\subsection{Neuron Cell Growth Experiment}

The neuron cells were prepared as described in 2.2. The cells were imaged using a 40x lens objective, with each $z$-stack consisting of 185 images $(74 \mu \mathrm{m})$. Although the density of the medium combined with the distance of the cells relative to 
the working distance of the lens caused a noticeable loss of focus throughout the $z$-stack, the findings were still useful for identifying proliferation along the nanofibers (Figure 9, Figure 10).

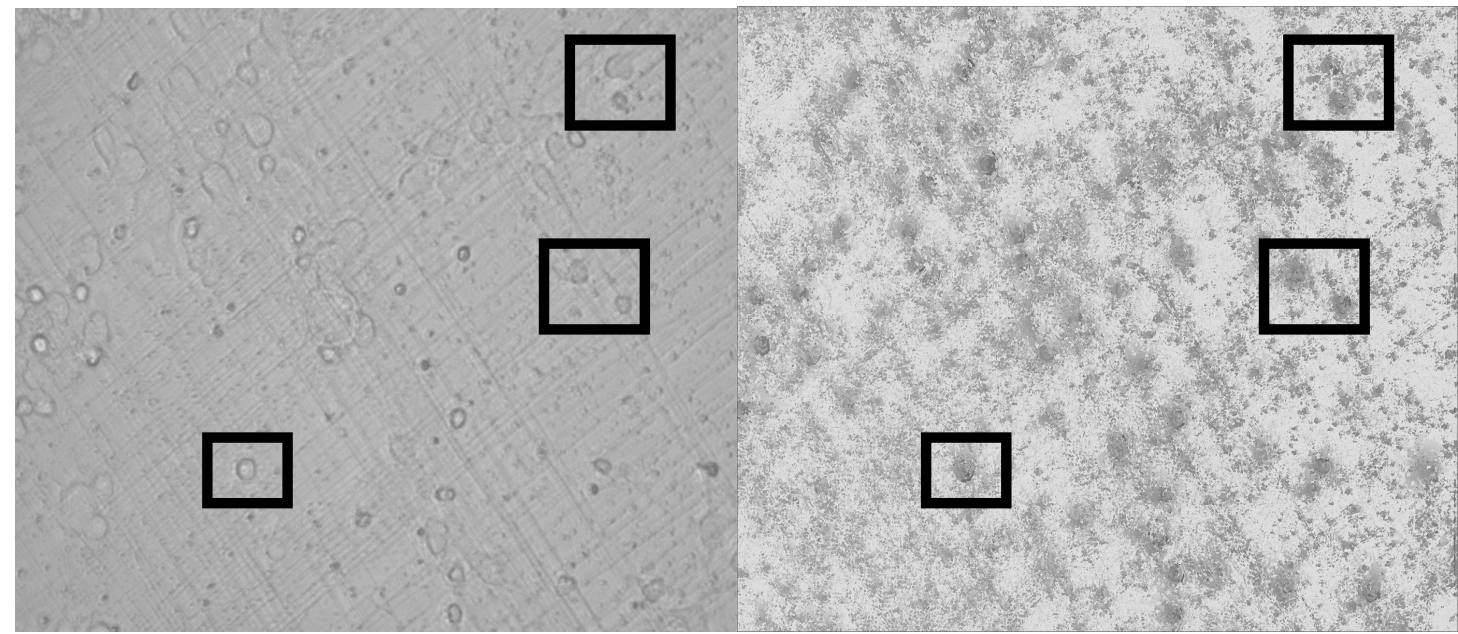

Figure 9: (Left) A z-stack image taken from the neuron sample and (right) the depth map of the region. Darker regions correspond to depth levels further up in the $z$-stack, and therefore higher up in the cell culture. Individual cells (highlighted) are identifiable within the depth map, demonstrating the potential tracking applications.

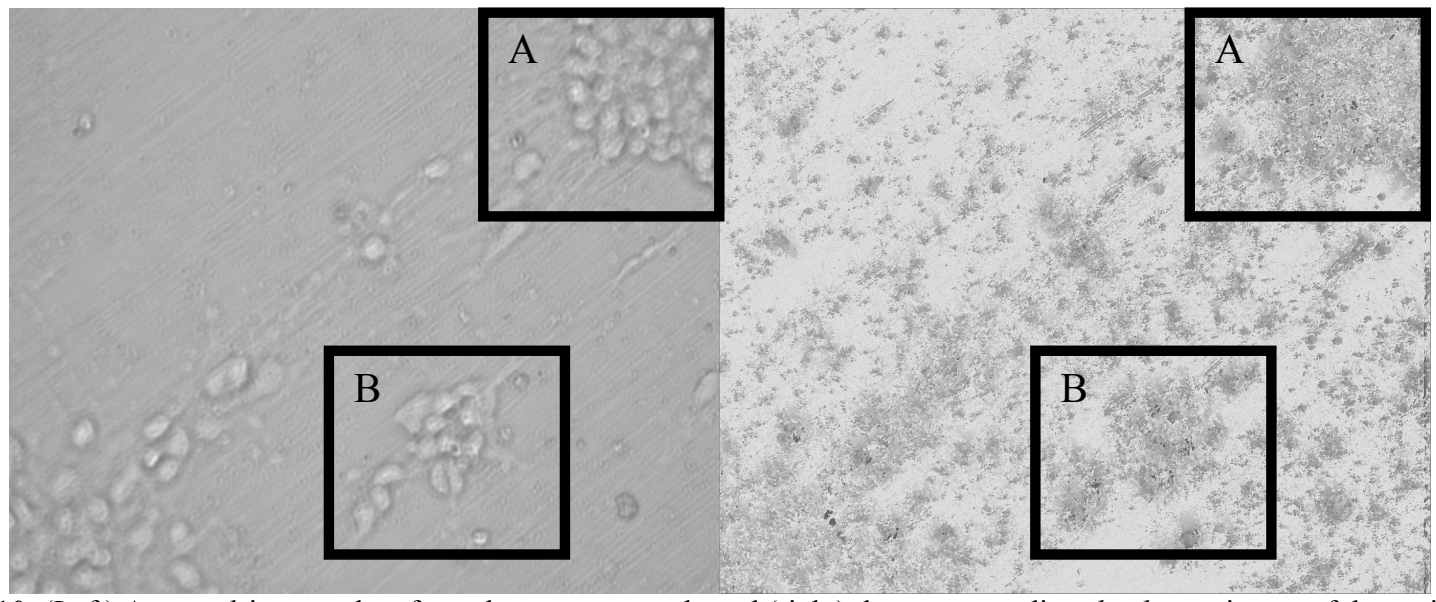

Figure 10: (Left) A $z$-stack image taken from the neuron sample and (right) the corresponding depth map image of the region. The clusters of cells in the image (A \& B) are distinguishable from the surrounding medium.

\section{DISCUSSIONS}

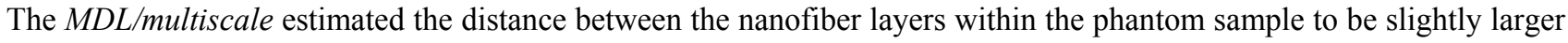
than the expected measurements. The discrepancy between the $40 \mathrm{x}$ and $20 \mathrm{x}$ lens measurements of the phantom sample is also notable, since the increased spatial resolution and finer $D O V$ when using the $40 \mathrm{x}$ lens magnification should offer a more accurate estimate than the 20x lens. This means that although the 20x lens gave a measurement closer to the theoretical one, it is possible that the z-resolution was insufficient to identify the true separation between the layers. Concerning the overall discrepancy between the estimated and expected distances there are a number of explanations that concern the assembly of the phantom sample. For example, the phantom sample may have minor spaces between the layers that would be physically impossible to remove by hand, particularly if the fibers themselves do not form a single layer. This may introduce an additional $1-3 \mu \mathrm{m}$ from overlapping fibers within the sample. The focus measures used identified upper nanofiber layer positions that corroborate with those estimated by the MDL/multiscale algorithm. The difficulty of identifying the lower nanofiber positions may be attributable to the fact that the mesh influences the focus measure of the image to a greater extent than the nanofibers. Since the focus measure is based on the entirety of the 
image, rather than localized regions of the nanofiber it is possible that a windowed approach could offer a more accurate measure of the lower nanofiber layer position.

With regards to the $M D L /$ multiscale performance, the information obtained concerning the locations of the nanofibers layers and cells demonstrates the potential for the tracking of fine structures within the sample without the usage of markers. Observations were made during the preliminary outgrowth experiment that will also be useful for future experiments. The usage of depth from delfocus techniques in the context of minimally manipulative imaging offers a viable solution to the problem of monitoring the behavior of live cells prior to their usage in cell therapies.

\section{CONCLUSION AND FUTURE WORK}

The application of the $M D L /$ multiscale demonstrated the potential of the technique in the label-free measurement of fine structures. The outcome of the neuron growth pilot study has provided a useful starting point for the development of an experimental framework for the investigation of label-free cell tracking. Based on the promising results of the phantom experiment, future work includes undertaking additional live cell studies to permit more detailed measurements of the cells and their locations.

\section{REFERENCES}

[1] Bernas T., Robinson J. P., Asem E. K., Rajwa B., "Loss of image quality in photobleaching during microscopic imaging of fluorescent probes bound to chromatin," J. Biomed. Opt. 10(6), 064015 (2005)

[2] Bkaily G., Pothier P., D'Orléans-Juste P., Simaan M., Jacques D., Jaalouk D., Belzile F., Hassan G., Boutin C., Haddad G., Neugebauer W., "The use of confocal microscopy in the investigation of cell structure and function in the heart, vascular endothelium and smooth muscle cells," Molecular and Cellular Biochemistry, 172, 171194, (1997)

[3] Burch C. R. and Stock J. P. P., "Some Applications of Phase-Contrast Microscopy," Quarterly Journal of Microscopical Science, 88(3), 491-499, (1947)

[4] Cremer C. and Cremer T. "Considerations on a laser-scanning-microscope with high resolution and depth of field" Acta Microscopica, 81(1), 31-44, (1978)

[5] Day R. N., Periasamy A., Schaufele F., "Fluorescence Resonance Energy Transfer Microscopy of Localized Protein Interactions in the Living Cell Nucleus," Methods, 25(1), 4-18, (2001)

[6] Eskicioglu, A.M.; Fisher, P.S., "Image quality measures and their performance," IEEE Transactions on Communications, 43(12), 2959-2965, (1995)

[7] H. Gu, Y. Shirai, and M. Asada, "MDL-based spatiotemporal segmentation from motion in a long image sequence," 1994 IEEE Computer Society Conference on Computer Vision and Pattern Recognition, 448-453, (1994)

[8] Hummel R. A., Kimia B., and Zucker S. W., "Deblurring Gaussian blur", Computer Vision, Graphics, and Image Processing, 38(1), 66-80, (1987)

[9] Namboodiri V.-P. and Chaudhuri S., "On Defocus, Diffusion and Depth Estimation," Pattern Recognition Letters, 28(3), 311-319, (2007)

[10] Smith W. A., Lam K.-P., and Richardson J. B., "2.5 D Scalable Reconstruction of Cell Cultures,” ISAP 2013, IEEE Signal Processing Society, (2013)

[11] Smith W. A., Lam, K.-P., Collins, D. J., and Tarvainen, J., "Estimation of Depth Map Using Image Focus: A Scale-Space Approach for Shape Recovery," Emerging Trends in Computing, Informatics, Systems Sciences, and Engineering, Springer New York, 1079-1090 (2013)

[12] Y. Sun, S. Duthaler and B. J. Nelson, "Autofocusing Algorithm Selection in Computer Microscopy," IEEE International Conference on Intelligent Robots and Systems, 70-76, (2005)

[13] Tarvainen J., Saarinen M., Laitinen J., Korpinen J., Viitanen J., "Creating Images with High Data Contents for Microworld Applications," Industrial Systems Review, 17-23, (2002)

[14] Yang Y., Wimpenny I., Ahearne M., "Portable nanofiber meshes dictate cell orientation throughout three dimensional hydrogels," Nanomedicine, 7(2), 131-6 (2011)

[15]Zernike, F. "Phase contrast, a new method for the microscopic observation of transparent objects part I," Physica 9(7), 686-698 (1942)

[16]Zernike, F. "Phase contrast, a new method for the microscopic observation of transparent objects part II," Physica 9(7), 686-698 (1942) 